\title{
Supersensitivity to Amphetamine in Protein Kinase-C Interacting Protein/HINTI Knockout Mice
}

\author{
Elisabeth Barbier', Agustin Zapata', Eric Oh², Qing Liu', Fei Zhu', Ashiwel Undie', Toni Shippenberg ${ }^{2}$ and \\ Jia Bei Wang*,I \\ 'Department of Pharmaceutical Sciences, School of Pharmacy, University of Maryland, Baltimore, MD, USA; ${ }^{2}$ Integrative Neuroscience Section, \\ NIDA Intramural Research Program, Baltimore, MD, USA
}

\begin{abstract}
Protein kinase $\mathrm{C}$ interacting protein/histidine triad nucleotide binding protein I (PKCl/HINTI) is a member of the histidine triad protein family. Although this protein is widely expressed in the mammalian brain including mesocorticolimbic and mesostriatal regions, its physiological function in CNS remains unknown. Recent microarray studies reported decreased mRNA expression of PKCI/HINTI in the frontal cortex of individuals with schizophrenia, suggesting the possible involvement of this protein in the pathophysiology of the disease. In view of the documented link between dopamine (DA) transmission and schizophrenia, the present study used behavioral and neurochemical approaches to examine the influence of constitutive PKCl/HINTI deletion upon: (i) basal and amphetamine (AMPH)evoked locomotor activity; (ii) DA dynamics in the dorsal striatum, and (iii) postsynaptic DA receptor function. PKCl/HINTI ${ }^{-/-}$(KO) mice displayed lower spontaneous locomotion relative to wild-type (WT) controls. Acute AMPH administration significantly increased locomotor activity in WT mice; nonetheless, the effect was enhanced in KO mice. Quantitative microdialysis studies revealed no alteration in basal DA dynamics in the striatum or nucleus accumbens of $\mathrm{KO}$ mice. The ability of acute AMPH to increase DA levels was unaltered indicating that function in presynaptic DA neurotransmission in these regions do not underlie the behavioral phenotype of $\mathrm{KO}$ mice. In contrast to WT mice, systemic administration of the direct-acting DA receptor agonist apomorphine $(10 \mathrm{mg} / \mathrm{kg})$ significantly increased locomotor activity in $\mathrm{KO}$ mice suggesting that postsynaptic DA function is altered in these animals. These results demonstrate an important role of $\mathrm{PKCl} / \mathrm{HINTI}$ in modulating the behavioral response to $\mathrm{AMPH}$. Furthermore, they indicate that the absence of this protein may be associated with dysregulation of postsynaptic DA transmission.

Neuropsychopharmacology (2007) 32, 1774- 1782; doi:I0.1038/sj.npp. I 30I30I; published online 3 January 2007
\end{abstract}

Keywords: schizophrenia; amphetamine-induced hyperactivity; dopamine; PKCl/HINTI

\section{INTRODUCTION}

Protein kinase $\mathrm{C}$ interacting protein/histidine triad nucleotide binding protein 1 (PKCI/HINT1) is an ubiquitous member of the histidine triad (HIT) protein family characterized by the presence of a conserved HIT (HisXHisXHis, $\mathrm{X}$ is a hydrophobic amino acid) sequence motif (Klein et al, 1998). The amino-acid sequences of the members of this family are conserved in a broad range of organisms including mycoplasm, plants, and mammals (Lima et al, 1996). Although murine PKCI/HINT1 is expressed in relatively high concentrations in brain, liver, and kidney, little is known about the physiological role of $\mathrm{PKCI} / \mathrm{HINT} 1$ proteins. Studies on the function of PKCI/

*Correspondence: Dr JB Wang, Department of Pharmaceutical Sciences, School of Pharmacy, University of Maryland, 20 North Pine Street, Baltimore, MD 21201, USA, Tel: + I 410706 6868, Fax: + I 4I0 706 0346, E-mail: jwang@rx.umaryland.edu

Received 24 May 2006; revised 27 September 2006; accepted 6 November 2006
HINT1 can only be found in a limited numbers of literatures. Bovine PKCI/HINT1 was originally identified as an inhibitor of PKC isoforms in vitro (McDonald and Walsh, 1985). However, the relevance of these in vitro effects to the physiological function of PKCI/HINT1 has been questioned (Klein et al, 1998). Studies in which X-ray crystallography and in vitro enzyme assays were used to elucidate the structure and function of PKCI/HINT1, suggested that it possesses nucleotidyl hydrolase or transferase activity (Lima et al, 1997). PKCI/HINT1 has also been shown to interact with the ataxia-telangiectasia group $\mathrm{D}$ protein and the $m i$ transcription factor in the yeast two-hybrid system (Brzoska et al, 1995). PKCI/HINT1 KO mice display increased susceptibility to carcinogenicity, suggesting that PKCI/HINT1 may normally play a tumor suppressor role (Su et al, 2003). Later, the protein has been reported to interact with pontin and reptin and inhibit TCF- $\beta$-catenin-mediated transcription (Weiske and Huber, 2005).

To date, only one study has examined the CNS actions of PKCI/HINT1. Using yeast two-hybrid screening, we recently 
showed that PKCI/HINT1 specifically interacts with the C-terminus of the mu opioid receptor (MOR) (Guang et al, 2004). This interaction led to an attenuation of receptor desensitization and inhibition of PKC-induced MOR phosphorylation. Furthermore, a deficiency in the expression of PKCI/HINT1 in mice enhanced both basal and morphine-induced antinociception. Morphine tolerance was also increased.

Recently, PKCI/HINT1 was identified as one of the candidate molecules in the neuropathology of schizophrenia via microarray analysis (Vawter et al, 2001, 2002). Expression of this gene was significantly decreased in cortical samples from patients with schizophrenia compared with age- and gender-matched controls. This change was subsequently validated by real-time quantitative polymerase chain reaction (PCR) (Vawter et al, 2004). Schizophrenia is a complex human disorder with unknown etiology. Several lines of evidence, however, suggest that increased dopaminergic neurotransmission may contribute to the pathophysiology of this disease (Seeman, 1987, Lewis and Lieberman, 2000). PET studies have revealed increased D2 receptor occupancy in patients with schizophrenia (AbiDargham et al, 2000) and DA receptor antagonists are used for the treatment of this disorder (Baldessarini and Tarazi, 2006). Amphetamine-evoked hyperactivity and stereotypy are used to model the positive symptoms of schizophrenia in rodents and reversal of these effects is considered as a desired property of antipsychotic drugs (Segal et al, 1981). This premise led us to hypothesize that PKCI/HINT1 may play a role in dopaminergic function. Accordingly, in the present study, WT mice and mice lacking the gene encoding PKCI/HINT1 protein were used to assess the involvement of PKCI/HINT1 in: basal and amphetamine (AMPH)-evoked locomotor activity, DA dynamics in the dorsal striatum, and postsynaptic DA receptor function. We also examined the distribution of PKCI/HINT1 in specific brain regions, including both mesocorticolimbic and mesostriatal areas, which are enriched in dopaminergic neurons.

\section{METHODS}

\section{PKCI/HINT1 ${ }^{-l-}$ Mice}

The generation of $\mathrm{PKCI} / \mathrm{HINT}^{-1-}$ mice was described previously and these mice have $96 \%$ of their genetic background from the 129 strain mice (Su et al, 2003). The breeding pairs of PKCI/HINT1 $1^{-1-}$ mice and their wild-type (WT) littermates were received from Dr IB Weinstein at Columbia University where the original $\mathrm{KO}$ mice were generated. Drug naïve animals used for the present study were homozygous mutant mice and WTs derived from homozygous mutant breeding pairs or WT breeding pairs, respectively. In addition, $\mathrm{KO}$ and their littermate controls were also used in AMPH $2.5 \mathrm{mg} / \mathrm{kg}$ experiments. The genotype of all progeny was confirmed by using PCR analysis of DNA extracted from tail biopsies. Animals were housed 4-5/cage and maintained under standard laboratory condition with food and water provided ad libitum. Male animals were tested between 10 and 20 weeks of age. WT and PKCI/HINT1 ${ }^{-1-}$ groups were matched for age in all experiments. All studies were conducted with an approved protocol of University of Maryland, School of Pharmacy
IACUC, or the IACUC of the NIH/NIDA IRP, and conformed to the NIH Guidelines for the Care and Use of Laboratory Animals.

\section{Locomotor Activity Measurement}

Basal and AMPH-evoked locomotor activity were monitored using a MED Associates' open-field activity monitoring system (Med Associates Inc., St Albans, VT). The chamber $(27 \times 27 \times 20.3 \mathrm{~cm})$ had sensor rings designed for use with mice. Each station has an on-board microprocessor to process beam breaks and prepare coordinate data for further analysis. Automated measurements of fine locomotion were previously validated (Sanberg et al, 1987). In our case, horizontal spontaneous locomotion activity, scored as ambulatory counts and stereotypic movements per $5 \mathrm{~min}$, configured according to the manufacturer's manual, were recorded during the first $30 \mathrm{~min}$ of acclimation to the novel environment and (1) for the consecutive $60 \mathrm{~min}$ of spontaneous activity or (2) for the consecutive $120 \mathrm{~min}$ following drug treatment. Stereotypic behavior is defined as small and fast movements occurring when the animal breaks the same beam pattern repeatedly. This beam pattern is a square of the size of the animal, each episode is separated by $100 \mathrm{~ms}$. Ambulation is defined as big and slow movements of the animal breaking a bigger beam pattern of two times the size of the animal. Data are expressed as total movement over the total recoded time. The animals were acclimated to a $12 \mathrm{~h}$ cycle light/dark cycle with the light on at 07:00 hours before the experiments. Dark phase experiments were conducted between 21:00 and 04:00 hours; all other tests were performed during the light phase of the cycle between 08:00 and 15:00 hours in the room where temperature was set at $23 \pm 2^{\circ} \mathrm{C}$. AMPH or other drugs were prepared freshly before each experiment by dissolving in saline $(\mathrm{NaCl} 0.9 \%)$ and administered via the intraperitoneal (i.p.) route in a $10 \mathrm{ml} / \mathrm{kg}$ volume of the indicated doses.

\section{In Vivo Microdialysis}

PKCI/HINT1 ${ }^{-1-}$ mice and their WT controls were anesthetized and implanted unilaterally with a microdialysis guide cannula (CMA/11, CMA microdialysis) in the nucleus accumbens (AP: $+1.5, \mathrm{~L}:-0.8, \mathrm{~V}:-3.8 \mathrm{~mm}$ from bregma) or the dorsal striatum (AP: $+0.4, \mathrm{~L}:-2.1, \mathrm{~V}:-2.2 \mathrm{~mm}$ from bregma) using standard stereotaxic techniques, and allowed to recover for 5 days before the microdialysis experiment as described (Chefer and Shippenberg, 2006). Twelve hours before the start of experiments, a microdialysis probe (CMA/11, 1 or $2 \mathrm{~mm}$ membrane length for accumbens and dorsal striatum, respectively, CMA microdialysis, North Chelmsford, MA) was connected to the dialysis system and flushed with artificial cerebrospinal fluid (aCSF: $145 \mathrm{mM}$ $\mathrm{NaCl}, 2.8 \mathrm{mM} \mathrm{KCl}, 1.2 \mathrm{mM} \mathrm{CaCl}, 1.2 \mathrm{mM} \mathrm{MgCl}_{2}, 0.25 \mathrm{mM}$ ascorbic acid, and $5.4 \mathrm{mM}$ D-glucose, $\mathrm{pH} 7.2$ adjusted with $\mathrm{NaOH} 0.5 \mathrm{M}$ ). The mouse was gently restrained and the probe was slowly inserted into the guide cannula. The dialysis system consisted of FEP tubing (CMA microdialysis) that connected the probe to a $1 \mathrm{ml}$ gastight syringe (Hamilton Co., Reno, NV) mounted on a microdialysis pump (CMA/102) through a quartz-lined, low-resistance 
swivel (375/D/22QM, Instech, Plymouth Meeting, PA). After probe insertion, the mouse was placed in the dialysis chamber with food and water freely available, and the probe perfused overnight with aCSF at a flow rate of $0.6 \mu \mathrm{l} / \mathrm{min}$. The next morning, the perfusion syringes were loaded with fresh aCSF and probes were allowed to equilibrate for an additional $1 \mathrm{~h}$ before the commencement of experiments. A flow rate of $0.6 \mu \mathrm{l} / \mathrm{min}$ was used for all studies.

For no net flux experiments, five different concentrations of DA $(0,5,10,20$, and $40 \mathrm{nM})$ in aCSF were perfused in random order through the dialysis probe. Each DA concentration was perfused for $30 \mathrm{~min}$, and then $2 \times 10 \mathrm{~min}$ samples were collected. Following completion of the no net flux experiments, normal aCSF was again perfused through the probe for $30 \mathrm{~min}$, allowing for a period of equilibration. Consecutive $15 \mathrm{~min}$ samples were then collected. After three baseline samples, mice received a saline i.p. injection and three more samples were collected. Then mice received a $2.5 \mathrm{mg} / \mathrm{kg}$ D-AMPH i.p. injection and samples were collected for an additional $90 \mathrm{~min}$. Samples were stored at $-80^{\circ} \mathrm{C}$ until analysis. The DA content was determined by HPLC coupled to electrochemical detection with external standards for quantification. All the samples were analyzed within $48 \mathrm{~h}$ of collection. After experiments, mice were killed by a pentobarbital overdose and their brains were removed, frozen on dry ice, and $20 \mu \mathrm{m}$ sections were obtained on a cryostat for the histological verification of probe location. No net flux data were analyzed as described (Chefer and Shippenberg, 2006). The net flux of DA through the probe (DAin-DAout) was calculated and plotted against the concentration of DA perfused (DAin). The following parameters were calculated from the resulting linear function. The $y$ axis intercept, corresponding to zero DA perfused through the probe is the dialysate DA concentration (DAdial) in a conventional microdialysis experiment. The $x$ axis intercept corresponds to the point of equilibrium where there is no net flux of DA through the probe and provides an unbiased estimate of extracellular DA concentration (DAext). The slope of the regression line corresponds to the extraction fraction (Ed) which has been shown to be an indirect measure of DA uptake (Smith and Justice, 1994; Chefer and Shippenberg, 2006). In the conventional microdialysis experiments investigating the effects of $\mathrm{AMPH}$, the average of the three baseline samples was calculated, and all the DA concentrations were expressed as \% of baseline. Differences between WT and KO animals in the appropriate variables were assessed by comparing both groups using a Student's $t$-test.

\section{Immunohistochemistry}

Animals and preparation of tissue: $\mathrm{PKCI} / \mathrm{HINT}^{-1-}$ and WT control adult mice were anesthetized with $7.5 \mathrm{mg}$ ketamine hydrochloride (Pfizer $\mathrm{AB}$, Sweden) and $2.5 \mathrm{mg}$ xylazine (Veterinaria AG, Switzerland) per $100 \mathrm{~g}$ body weight i.p. Animals were perfused transcardially with saline followed with $4 \%$ paraformaldehyde (PFA) in phosphate buffer $(0.1 \mathrm{M}, \mathrm{pH} 7.4)$ for $10 \mathrm{~min}$. The whole brain was removed, postfixed in $4 \%$ PFA at $4{ }^{\circ} \mathrm{C}$ overnight, and equilibrated with $30 \%$ sucrose in phosphate buffer at $4{ }^{\circ} \mathrm{C}$ for $48 \mathrm{~h}$. Embedded whole brain (OCT, Tissue-Tek, Sakura Finetek
USA Inc.) was cut coronally into $25 \mu \mathrm{m}$ sections using a cryostat (OTF5000, Bright, Jencons, UK). All sections were kept with long-term protecting solution at $4^{\circ} \mathrm{C}$ until used.

Floating sections were incubated with $1 \%$ hydrogen peroxide in $70 \%$ methanol-tris buffered saline (TBST; $0.1 \mathrm{M}$ Tris, $\mathrm{pH} 7.4,0.9 \%$ saline, and $0.3 \%$ Triton $\mathrm{X}-100$ ) for $30 \mathrm{~min}$ at room temperature (all incubations were performed at $22-25^{\circ} \mathrm{C}$ ) to inhibit endogenous peroxidase, then washed three times with TBST and incubated for $1 \mathrm{~h}$ in $1 \%$ bovine serum albumin (BSA) - TBST. For staining, the sections were incubated with hPKCI antiserum $(1: 10000$ dilution in TBST, $1 \%$ BSA, $24 \mathrm{~h}$ ), washed in TBST (three times of $5 \mathrm{~min}$ duration each), incubated for $1 \mathrm{~h}$ in biotinylated donkey anti-rabbit serum $(1: 1000$ dilution; Biotin-SP-Conjugated Donkey Anti-Rabbit IgG $(\mathrm{H}+\mathrm{L})$, Jackson ImmunoResearch Lab Inc., West Grove, PA, USA), rinsed, and finally visualized with $A B C$ reagent (ABC Kit PK-6100, Vector, USA) according to manufacturer's manual and mounted onto gelatin-coated slides in DPX mounting medium.

Slides were viewed using Nikon E800 microscope, images were captured with Nikon digital camera, and edited using Photoshop (v CS; Adobe Systems Inc., San Jose, CA, USA).

For immunofluorescence staining, sections were incubated for $1 \mathrm{~h}$ at room temperature with $1 \%$ BSA $-1 \%$ normal donkey serum in Tris-buffered saline (TBST; $0.1 \mathrm{M}$ Tris, $\mathrm{pH} 7.4,0.9 \%$ saline, and $0.3 \%$ Triton $\mathrm{X}-100$ ), then for $24 \mathrm{~h}$ in hPKCI antiserum ( $1: 1000$ dilution in $1 \% \mathrm{BSA} /$ TBST), washed in TBST (three times of $5 \mathrm{~min}$ each), followed by $1 \mathrm{~h}$ incubation with Cy2-conjugated donkey anti-rabbit antibody (1:500 dilution; Jackson ImmunoResearch Lab Inc., West Grove, PA, USA). After wash, they were incubated for $24 \mathrm{~h}$ in a mixture of mouse anti-NeuN $(1: 1000$ dilution in $1 \%$ BSA/TBST; Neuronal Nuclei, Chemicon) and goat anti-GFAP (1:500 dilution in 1\% BSA/TBST; glial fibrillary acidic protein, Santa Cruz Biotechnology Inc.) and washed with TBST as above. The sections were then incubated with a mixture of Cy5conjugated donkey anti-mouse antibody and Cy3-conjugated donkey anti-goat antibody (1:500 in 1\% BSA/TBST; Jackson ImmunoResearch Lab Inc., West Grove, PA) for $60 \mathrm{~min}$, washed in TBST as above, incubated with $2 \mathrm{nM}$ DAPI (4'-6-diamidino-2-phenylindole, Molecular Probes) for $60 \mathrm{~min}$, washed in TBS, and mounted. Slides were viewed under Nikon E800 microscope and images were captured on a Fluo View X confocal microscope (Olympus Instruments, CA, USA).

\section{Statistical Analysis}

Two-way analyses of variance (ANOVA) of genotype $\times$ phase and genotype $\times$ treatment were used to compare the results of the behavioral experiments. When ANOVA was significant $(p<0.05)$, post hoc comparisons between groups were made using Bonferroni test. Comparison of the parameters derived from the no net flux analysis was performed by a Student's $t$-test. Data are presented as mean \pm SEM. Statistical analyses were performed using Graphpad Prism version 4.00 for Windows (Graphpad Software, San Diego, CA, USA). 


\section{RESULTS}

\section{PKCI/HINT1 ${ }^{-I-}$ Mice Display Decreased Spontaneous Locomotor Activity and Supersensitive Response to Amphetamine}

Spontaneous locomotor activity measured during the light phase of the cycle (between 08:00 and 15:00 hours) and during the dark phase of the cycle (between 21:00 and 04:00 hours) which corresponds to the active phase, is shown in Figure 1. During the light phase, WT and KO mice displayed a lower level of spontaneous activity, measured as ambulatory and stereotypic movement for $60 \mathrm{~min}$. In the dark phase, both genotypes exhibited an increase of locomotion as expected (light phase $v s$ dark phase: WT: $p<0.0001$ for acclimation and spontaneous; KO: $p<0.0001$ for acclimation and $p<0.01$ for spontaneous; Bonferroni). However, the KO mice consistently scored on average $40 \%$ lower than the WT in either the light or the dark phase (WT vs KO: stereotypy: $p<0.01$ light phase acclimation and dark phase spontaneous; ambulation: $p<0.0001$ dark phase acclimation and spontaneous; Bonferroni). These results indicate that the $\mathrm{KO}$ mice are hypolocomotive when tested a

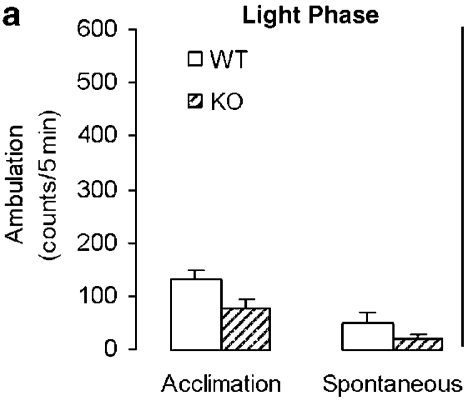

b

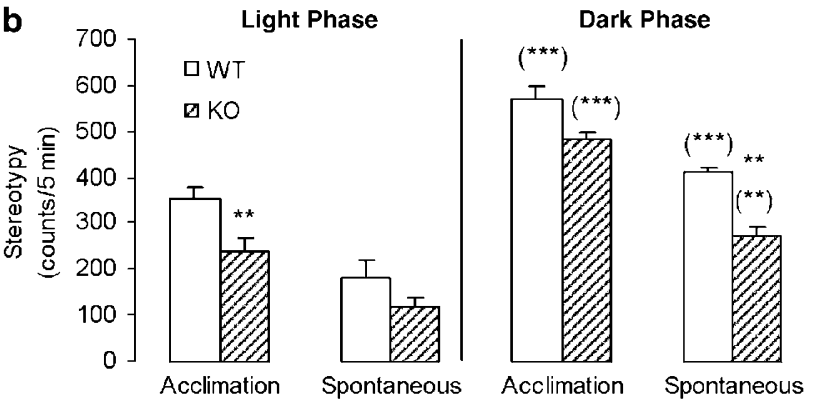

Figure I Spontaneous locomotion activity in the WT and PKCl/ $\mathrm{HINTI}^{-l-}$ mice during light/dark phase of the cycle. Locomotion was monitored during the $30 \mathrm{~min}$ of acclimation to the novel environment followed by a I h period. Counts per $5 \mathrm{~min}$ were averaged over the $30 \mathrm{~min}$ of acclimation and the consecutive I h of spontaneous activity; mean \pm SEM are represented. (a) Ambulation during acclimation (for phase, $F_{(1.26)}=1 \mid 9.21, p<0.000 \mathrm{I}$; for genotype, $F_{(1.26)}=30.07, p<0.000 \mathrm{I}$; for interaction $\left.F_{(1,26)}=9.47, p=0.0049\right)$ and during the following $\mathrm{I} h$ of spontaneous activity (for phase, $F_{(1,26)}=78.53, p<0.000$ I; for genotype, $F_{(1,26)}=39.84, p<0.000$ I; for interaction $\left.F_{(1,26)}=17.94, p=0.0003\right)$. (b) Stereotypy during acclimation (for phase, $F_{(I .26)}=44.85, p<0.0001$; for genotype, $F_{(1,26)}=12.77, p=0.0014$; for interaction $F_{(1,26)}=0.29$, $p=0.5937$ ) and during the following $\mathrm{I} h$ of spontaneous activity (for phase, $F_{(I, 26)}=81.55, p<0.0001$; for genotype, $F_{(I, 26)}=16.13, p=0.0004$; for interaction $\left.\mathrm{F}_{(1,26)}=1.74, p=0.211 \mathrm{I}\right)$. $^{* * *} p<0.01$ and ${ }^{(* * *)} p<0.000 \mathrm{I}$ compare dark phase vs light phase; $p<0.01$, **** $p<0.000$ I compare WT vs $\mathrm{KO}$; WT and $\mathrm{KO}$ light phase $n=9$; $\mathrm{WT}$ and $\mathrm{KO}$ dark phase $n=6$; two-way ANOVA genotype $x$ phase, followed by Bonferroni. under habituated basal conditions (light/dark phases) or during exploration of a novel environment (acclimation phase) in comparison with the WT mice.

Rodent locomotor activity is known to be affected by CNS stimulants. The effect of AMPH on the locomotor activity of PKCI KO mice was examined in this study. As shown in Figure 2, AMPH (NIDA drug supply program) at a dose of $2.5 \mathrm{mg} / \mathrm{kg}$ increased locomotion in both WT and $\mathrm{KO}$ (ambulation: $p<0.0001$ vs saline in KO; stereotypy: $p<0.01$ vs saline in WT, $p<0.0001$ vs saline in KO; Bonferroni). However, AMPH-evoked locomotor activity was significantly enhanced ( $\geqslant 3$-fold) in PKCI KO mice (WT $v s$ KO: $p<0.0001$ for ambulation and stereotypy). To determine whether this enhanced response was selective or occurred in response to other drug of abuse, locomotor activity in response to the MOR agonist morphine was assessed; the response to the GABA antagonist bicuculline was also examined. Morphine (NIDA drug supply program) and bicuculline (RBI, Natick, MA, USA) at a dose of 10 and $1 \mathrm{mg} / \mathrm{kg}$, respectively, did not promote increase in locomotor activity as shown in Figure 2. The increase of AMPHevoked activity in the KO mice was observed over a range of 

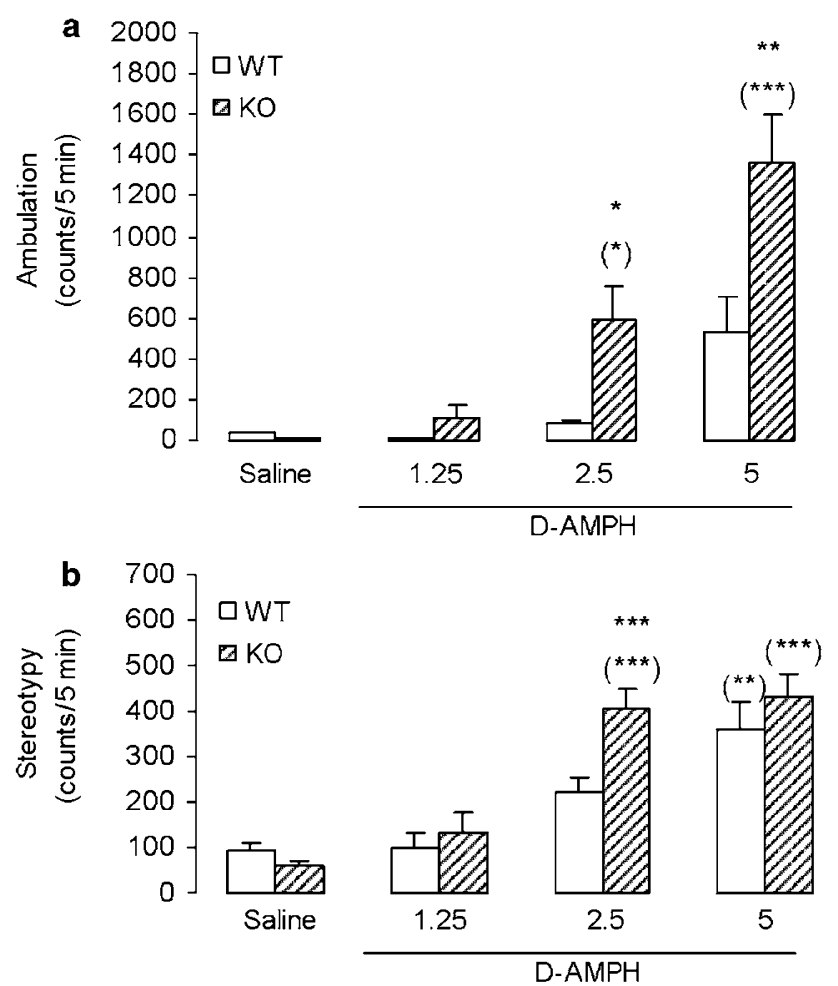

Figure 3 Dose-response to acute D-amphetamine in $\mathrm{WT}$ and $\mathrm{PKCl} /$ $\mathrm{HINTI}^{-1-}$ mice. Saline $(10 \mathrm{ml} / \mathrm{kg}$ i.p.) or D-AMPH $(1.25,2.5$, and $5 \mathrm{mg} / \mathrm{kg}$ i.p.) was administered after the period of acclimation and locomotion was measured during the following $120 \mathrm{~min}$. (a) Ambulation (for treatment $F_{(3,57)}=15.44, p<0.0001$; for genotype $F_{(1,57)}=10.79, p=0.0018$; for interaction $F_{(3,57)}=3.25, p=0.0284$ ). (b) Stereotypy (for treatment $F_{(3,57)}=24.75, \quad p<0.0001$; for genotype $F_{(1,57)}=7.02, p=0.01$; for interaction $\left.F_{(3.57)}=4.15, p=0.0099\right)$. $\left(^{*}\right) p<0.05, \quad(* *) p<0.01$, and $(* * * *) p<0.000$ I compare saline vs $\mathrm{AMPH}$; $* p<0.05$, *** $p<0.01$, and **** $p<0.000$ I compare WT vs KO. Saline and AMPH I.25 mg/kg WT and $\mathrm{KO} n=6 ; \mathrm{AMPH} 2.5 \mathrm{mg} / \mathrm{kg} \mathrm{WT}=12$ and $\mathrm{KO}=14$ and $\mathrm{AMPH} 5 \mathrm{mg} / \mathrm{kg}$ WT $n=6$ and $K O n=9$; two-way ANOVA genotype $\times$ treatment, followed by Bonferroni.

AMPH doses as shown in Figure 3 (ambulation in KO: $p<0.05$ for AMPH $2.5 \mathrm{mg} / \mathrm{kg} v s$ saline, $p<0.001$ for AMPH $5 \mathrm{mg} / \mathrm{kg} v s$ saline; Bonferroni). To determine whether the enhancement of activity could be due to a decrease in stereotypy, this behavior was also assessed simultaneously. Similarly, AMPH produces a dose-related increase in stereotypy (stereotypy in KO: $p<0.0001$ for AMPH 2.5 and $5 \mathrm{mg} / \mathrm{kg}$ vs saline; Bonferroni). As shown in Figures 2 and 3, lack of PKCI/HINT1 is associated with an increase in both AMPH-evoked ambulation (WT $v s$ KO: $p<0.05$ for AMPH $2.5 \mathrm{mg} / \mathrm{kg} ; p<0.01$ for AMPH $5 \mathrm{mg} / \mathrm{kg}$; Bonferroni) and stereotypy (WT $v s$ KO: $p<0.0001$ for AMPH $2.5 \mathrm{mg} / \mathrm{kg}$; Bonferroni).

Amphetamine increases extracellular DA levels by stimulating its release and by reversing DA transport. Interactions of AMPH with other biogenic amines have also been reported. Therefore, in order to probe specifically the DA system, additional studies were conducted to determine whether PKCI/HINT1 deletion alters the locomotor activating effects of the selective DAT inhibitor, GBR 12909 (TOCRIS, Ellisville, MI, USA). Analogous to AMPH, a significant enhancement of GBR 12909-evoked locomotion a
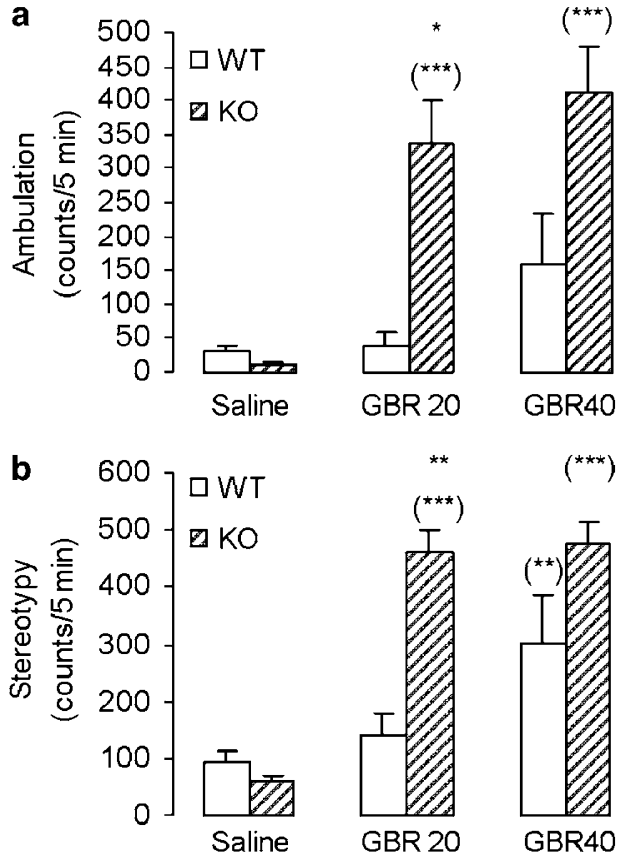

Figure 4 Dose-response to acute GBR 12909 in WT and $\mathrm{PKCl} /$ $\mathrm{HINTI}^{-1-}$ mice on locomotion. Saline (I 0 ml/kg i.p.) or GBRI2909 (20 and $40 \mathrm{mg} / \mathrm{kg}$ i.p.) was administered after the period of acclimation and locomotion was measured during the following $120 \mathrm{~min}$. (a) Ambulation (for treatment $F_{(2,38)}=11.35, p<0.000 \mathrm{I}$; for genotype $F_{(1,38)}=14.67$, $p=0.0005$; for interaction $F_{(2,38)}=4.6, p=0.0162$ ). (b) Stereotypy (for treatment $F_{(2,38)}=25.22, \quad p<0.000 \mathrm{I}$; for genotype $F_{(1,38)}=17.97$, $p=0.000 \mathrm{I}$; for interaction $\left.F_{(2,38)}=7.82, p=0.0014\right)$. ${ }^{(* *)} p<0.01$ and (***) $^{*}<0.000$ I compare saline vs GBR; $* p<0.05$ and $* * * 0.01$ compare WT vs $K O ; n=7$ for $W T$ and $n=9$ for $K O$; two-way ANOVA genotype $\times$ treatment, followed by Bonferroni.

was seen from the KO mice. As shown in Figure 4, GBR 12909 elicited an increase in locomotion in both WT and $\mathrm{KO}$ mice at a dose of $40 \mathrm{mg} / \mathrm{kg} \quad(p<0.0001$ vs saline; Bonferroni). The dose of $20 \mathrm{mg} / \mathrm{kg}$ induced hyperlocomotion only in the KO animals (WT $v s$ KO: $p<0.05$ for ambulation, $p<0.01$ for stereotypy; Bonferroni). These results indicate that mice without PKCI/HINT1 are supersensitive to AMPH and this enhanced response is likely mediated through the dopaminergic system, as it is mimicked by a selective DA transporter blocker.

\section{Extracellular DA Levels in Nucleus Accumbens and Dorsal Striatum}

DA projections to the striatum play an important role in the control of locomotor activity. Therefore, we used the technique of in vivo microdialysis in order to investigate the consequences of genetic deletion of the PKCI/HINT1 protein on basal extracellular DA dynamics as well as on AMPH-evoked alterations in DA levels of dialysate. Quantitative no net flux microdialysis revealed no significant differences in basal dialysate (DAdial) levels or in the estimated DAext) in the nucleus accumbens (Figure 5, $t=-0.47, \mathrm{df}=16, p=0.65$ and $t=0.04, \mathrm{df}=16, p=0.97$ for DAdial and DAext, respectively). Moreover, the DA extraction fraction (Ed), calculated as the slope of the no net flux regression line was unchanged in $\mathrm{KO}$ mice $(t=0.15, \mathrm{df}=16$, 
$p=0.88$ ), suggesting that deletion of the PKCI/HINT1 did not alter the clearance of extracellular DA by the DA transporter (Smith and Justice, 1994; Chefer and Shippenberg, 2006). Similar negative results were obtained in the caudate putamen (DAdial: $t=0.58, \mathrm{df}=10, p=0.58$; DAext: $t=1.01, \mathrm{df}=10, p=0.33$; Ed: $t=0.81, \mathrm{df}=10, p=0.43)$. Conventional microdialysis revealed a marked increase in basal dialysate DA levels in response to AMPH. No significant difference between $\mathrm{WT}$ and $\mathrm{KO}$ mice in AMPH-evoked DA levels was observed in the dorsal striatum $(t=0.58, \mathrm{df}=10, p=0.58)$. The maximal AMPH response was $1024 \pm 104$ and $1103 \pm 89 \%$ of basal levels in WT and KO mice, respectively. Similarly, no genotype difference was observed in the nucleus accumbens (maximal AMPH response $405 \pm 73$ vs $403 \pm 47 \%$ of baseline in WT and KO mice, respectively, $t=0.02, \mathrm{df}=16, p=0.98$ ). As the dose of AMPH used in microdialysis studies was that
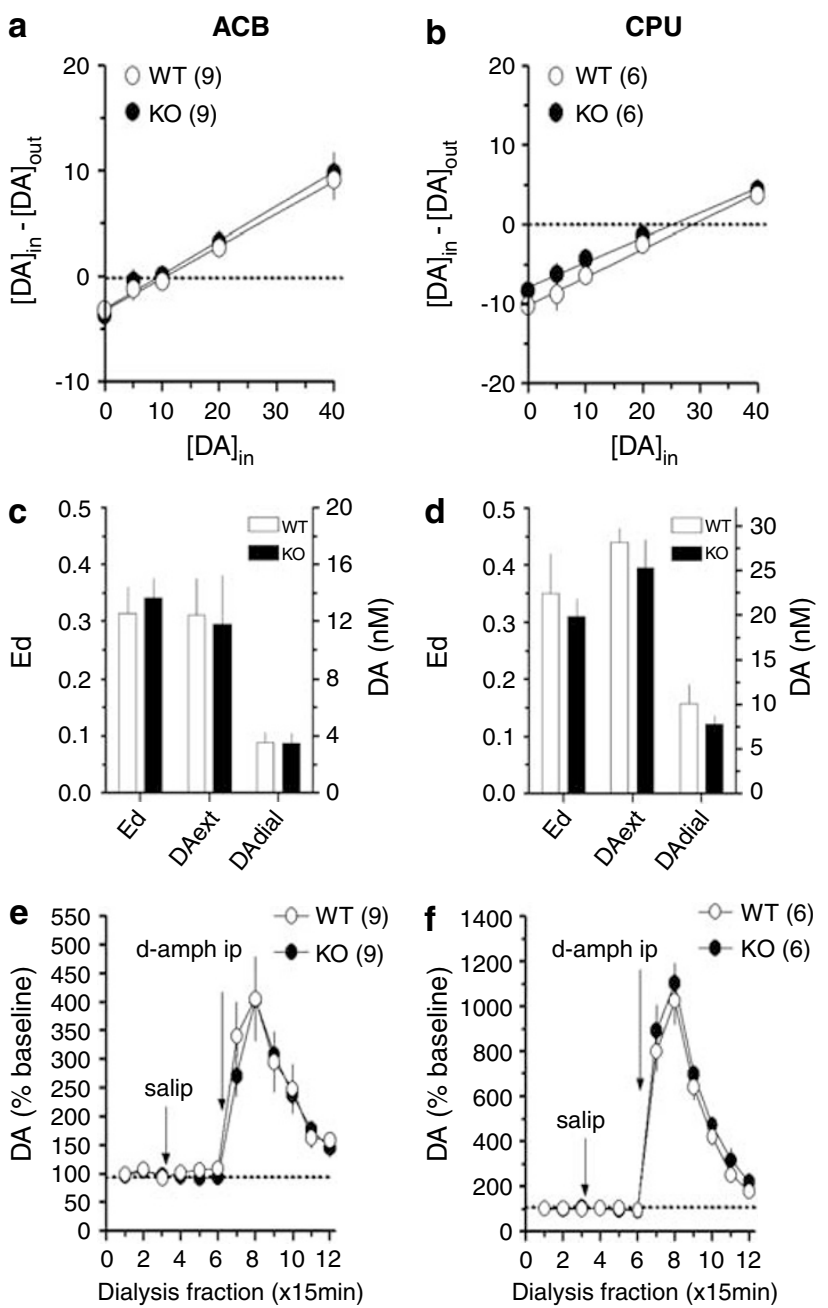

Figure 5 Basal and amphetamine-evoked extracellular DA in the nucleus accumbens and caudate-putamen of $\mathrm{WT}$ and $\mathrm{PKCl} / \mathrm{HINTI}{ }^{-1-}$ mice. No net flux microdialysis was used to asses the status of extracellular DA in the basal state. No significant genotype differences were observed in the estimated DAext $(a, b)$ nor in the extraction fraction (Ed; $c, d)$ suggesting that both release and uptake of DA are unchanged in the KO mice. In addition the amphetamine-evoked DA response did not differ in WT and $\mathrm{KO}$ animals (e, f). The same results were obtained in both, the nucleus accumbens ( $a, c$, and e) and the caudate-putamen (b, $d$, and $f$ ). producing an enhanced locomotor response in KO mice, the lack of genotype differences in the AMPH-evoked DA response suggests that the enhanced behavioral sensitivity to AMPH is due to changes at the postsynaptic rather than presynaptic level.

\section{Apomorphine Induced a Differential Hyperlocomotor Activity in PKCI/HINT1 ${ }^{-I-}$ Mice}

To further explore the mechanism responsible for the behavioral supersensitivity to AMPH observed in the KO mice, we tested the locomotor response to the nonselective DA receptor agonist, apomorphine. A high dose $(10 \mathrm{mg} / \mathrm{kg})$ was used in order to probe postsynaptic DA receptor function. Data shown in Figure 6 revealed that the KO mice exhibited significantly higher (two- to threefold) locomotor activity as compared to WT in both total ambulation $(p<0.01$; Bonferroni) and stereotyped behavior $(p<0.0001$; Bonferroni). This result indicates that PKCI/HINT1 deletion is associated with postsynaptic DA receptor supersensitivity.

\section{PKCI/HINT1 Brain Distribution and Neuronal Expression}

Western blotting was used to determine the expression of PKCI/HINT1 in the cortex, cerebellum, midbrain, and
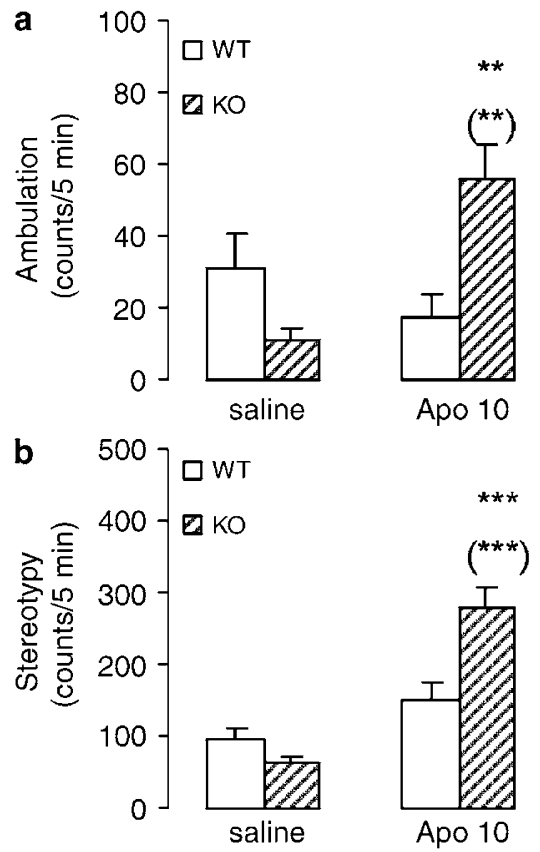

Figure 6 Apomorphine-induced hyperlocomotion in $\mathrm{PKCl} / \mathrm{HINTI}^{-1-}$ mice. Saline ( $10 \mathrm{ml} / \mathrm{kg}$ i.p.) or apomorphine ( $10 \mathrm{mg} / \mathrm{kg}$ i.p.) was administered after the period of acclimation and locomotion was measured during the following $120 \mathrm{~min}$. Results are expressed as mean \pm SEM of total scores. (a) Ambulation (for treatment $F_{(1,18)}=4.26, p=0.0538$; for genotype $F_{(1,18)}=1.55, p=0.2296$; for interaction $\left.F_{(1,18)}=14.6, p=0.0013\right)$. (b) Stereotypy (for treatment $F(1,18)=49.32, p<0.0001$; for genotype $F_{(1,18)}=5.91, p=0.0257$; for interaction $\left.F_{(1,18)}=16.51, p=0.0007\right)$. (**) $p<0.01$ and ${ }^{(* * *)} p<0.000$ I compares saline vs Apo; *** $p<0.01$ and **** $p<0.000$ I compares WT vs KO. Saline WT and KO $n=6$; APO WT and $\mathrm{KO} n=5$; two-way ANOVA genotype $\times$ treatment, followed by Bonferroni. 


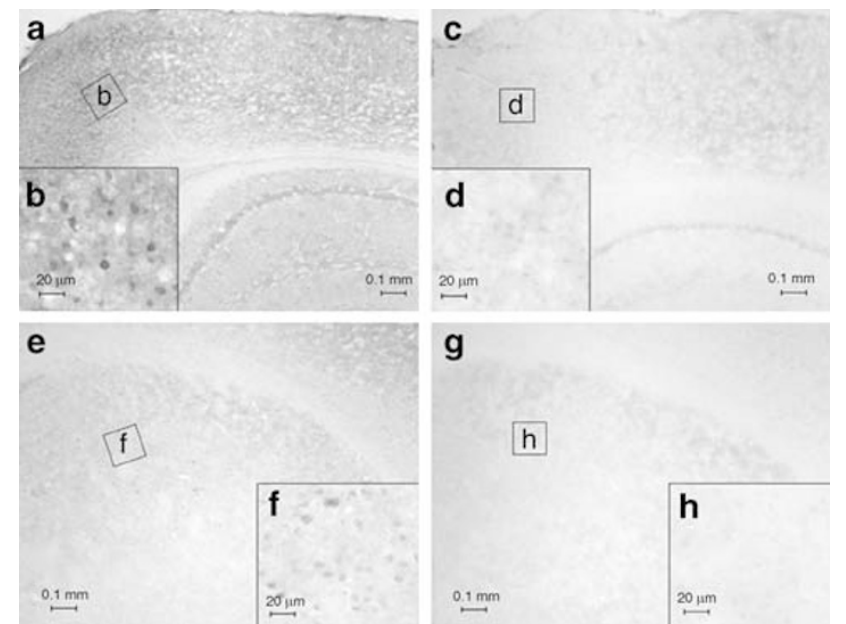

Figure $7 \mathrm{PKCl} / \mathrm{HINTI}$ immunoreactivity in mouse brain region of frontal cortex and caudate putamen (striatum). (a, b and e, f) WT mouse; (c, d and $g$, h) $\mathrm{KO}$ mouse. PFA-fixed frozen brain coronal sections were treated with rabbit anti-PKCl polyclonal antiserum, dilution 1:10000, followed by biotin-SP-conjugated donkey anti-rabbit IgG, dilution I : I000. Dark blue staining represents $\mathrm{PKCl} / \mathrm{HINTI}$ positive cells. $(a-d)$ frontal cortex; (e-h) caudate putamen (striatum).

spinal cord of KO and WT mice (data not shown). These experiments revealed a broad expression pattern of PKCI/ HINT1 in brain and spinal cord of mice, suggesting an important role for PKCI/HINT1 in neurological system. In contrast, no expression was observed in KO mice confirming the absence of this gene. Immunohistochemical analysis of PKCI staining in WT mice revealed a localization pattern consistent with neurons and neuronal processes in various brain regions including frontal cortex and striatum (Figure 7). There was only low-level background staining in brain sections from PKCI/HINT1 gene knockout mice (Figure 7). PKCI/HINT1 staining could be clearly visualized within neurons and neuronal processes in quad-labeled fluorescent PFA-fixed frozen sections from various brain regions. A representative picture taken from thalamus is presented in Figure 8. PKCI/HINT1 immunoreactivity (green) was observed in neurons labeled with specific antineuronal marker antibody (anti-NeuN; blue), but was absent from astrocytes stained with specific anti-glia marker antibody (anti-GFAP; red). Although a low level of nonspecific background reactivity was noted as a light green color in sections from both WT and KO mice, this did not interfere with the observation of specific immunoreactivity.

\section{DISCUSSION}

The present studies demonstrate an important role of PKCI/HINT1 in modulating basal locomotor activity and the behavioral response to AMPH.

Furthermore, they suggest that lack of PKCI/HINT1 is associated with dysregulation of postsynaptic DA transmission.

The KO mice displayed decreased basal locomotor activity compared to WT controls both in a novel environment and after habituation. However, D-AMPH-
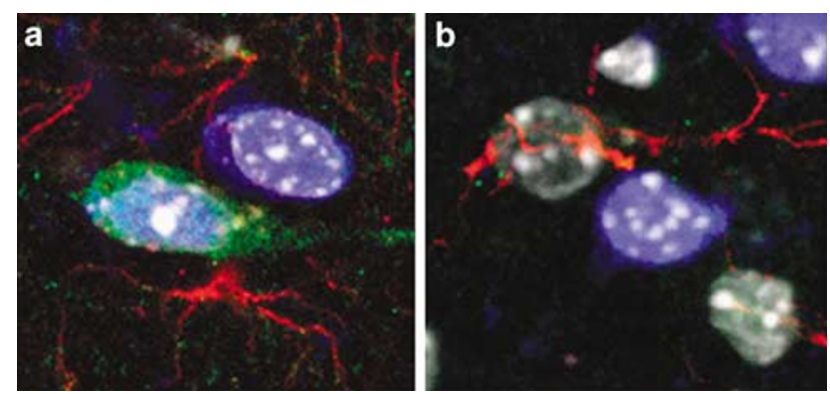

Figure 8 Quad-labeling in PFA-fixed frozen brain sections. (a) WT mouse; (b) $\mathrm{KO}$ mouse. PKCl/HINTI was detected with anti-hPKCl polyclonal antiserum plus Cy2-conjugated donkey anti-rabbit antibody (green); neurons were identified with anti-NeuN monoclonal IgG plus Cy5conjugated donkey anti-mouse antibody (blue); glial cells were identified with anti-GFAP polyclonal antiserum plus Cy3-conjugated donkey anti-goat antibody (red); and DAPI (white)-detected DNA in nuclei.

induced locomotor activity in $\mathrm{KO}$ mice is substantially higher than in WT animals suggesting that lack of functional PKCI/HINT1 protein results in hypersensitivity to the psychostimulant effects of AMPH. The lack of differences in locomotor activity in response to morphine and bicuculline also indicates that the enhanced locomotor activity in the KO is specific to AMPH. It is known that central DA systems are involved in the initiation and ongoing control of locomotion. The psychostimulant AMPH produces profound motor activation and this property is mediated at least in part by promoting the release of DA from presynaptic vesicles, as well as norepinephrine and serotonin. The primary targets of AMPH in the CNS are the monoamine transporters. Thus, specific monoamine transporter inhibitors will be useful tools to probe which of the three monoamines is responsible for enhanced LMA. In this study, a DA transporter-specific inhibitor, GBR 12909, induced a similar response of LMA in the PKCI/HINT1 KO mice as $\mathrm{AMPH}$, therefore, demonstrated that $\mathrm{DA}$ is the most likely neurotransmitters involved in AMPH-induced LMA in our study.

AMPH-induced release of DA through the DA transporter (Sulzer et al, 1993; Schenk, 2002) is critical for AMPHinduced behavioral activation as mice lacking the DA transporter are insensitive to the locomotor stimulant effects of AMPHs (Giros et al, 1996). Thus, the enhanced behavioral sensitivity to $\mathrm{AMPH}$ in the $\mathrm{KO}$ mice might be explained by an enhanced AMPH-evoked DA overflow in $\mathrm{NAc}$ and $\mathrm{CPu}$. However, the in vivo microdialysis data from this study did not support this possibility; there was no significant difference between WT and KO mice in AMPHevoked DA levels observed in either the dorsal striatum or nucleus accumbens, suggesting that increased presynaptic DA release is not likely the cause for the behavioral supersensitivity to AMPH observed in the KO mice. Other explanations for this supersensitive behavior need to be explored. Apomorphine is a directly acting DA agonist. Low dose of apomorphine activates the presynaptic autoreceptors and decreases postsynaptic activity, whereas higher dose increase locomotion by activating postsynaptic DA receptors. In the present study, a relatively high dose of apomorphine $(10 \mathrm{mg} / \mathrm{kg})$ resulted in an enhanced locomotion in the KO mice. As previously reported (Halberda et al, 
1997), selectively activation of D1- or D2-like receptors would produce different locomotor activity on mice. As a nonselective DA receptor agonist, apomorphine induced a modest increase of locomotor activity on the mPKCI KO mice but no change in control animals. This may well reflect a combined effect of apomorphine on both D1- and D2-like receptors.

Our findings from the LMA study, together with the results of the microdialysis study, suggest that changes at the postsynaptic level in the KO mice are more likely responsible for the observed increased sensitivity to AMPH. However, the specific postsynaptic modifications in the $\mathrm{KO}$ mice remain to be determined. Changes in the expression of DA receptors, alterations in receptor signal transduction, modification of the receptor phosphorylation, all can lead to functional consequences similar to what was observed in this study. Indeed, our laboratory results of GTP $\gamma s$ binding assay using the $\mathrm{KO}$ mice brain tissues has indicated that both D1- and D2-like receptor activities are enhanced in the KO mice (unpublished data). Results from our previous study (Guang et al, 2004) also showed that PKCI/HINT1 inhibited PKC-related MOR phosphorylation. It is not clear whether the inhibition of MOR phosphorylation in PKCI/ HINT1-expressing cells is due to direct inhibition of PKC activity or other alternative mechanism. Nevertheless, this result indicated that $\mathrm{PKCI} / \mathrm{HINT} 1$ could play an important role in the regulation of phosphorylation of neurotransmitter receptors. Previous studies suggested that PKC-mediated phosphorylations of $D_{1}$ and $D_{2}$ receptors were involved in receptor internalization (Namkung and Sibley, 2004) and this rapid receptor sequestration is involved in agonistinduced receptor desensitization. Thus, PKCI/HINT1 may affect the postsynaptic sensitivity by altering the receptor phosphorylation state and/or interfering with receptor desensitization.

The existence of a tonically active MOR system that stimulates mesoaccumbems DA neurotransmission is well established (Spanagel et al, 1992; Herz, 1998). Our previous study had revealed that PKCI/HINT1 could attenuate PKCmediated phosphorylation of MOR and regulate its function (Guang et al, 2004). The possibility exists that changes in opioid function in the PKCI/HINT1 KO can contribute to the supersensitivity to AMPH observed in these animals in the present study. Surprisingly, however, PKCI/HINT1 KO mice did not show increased sensitivity to morphineinduced locomotor activation. Therefore, this result seems not supporting the opioid involvement. However, it may also be explained by the strain variation among different mice as both stimulatory and depressive locomotor effects of morphine have been reported depending on the dose and the interval after the administration (Patti et al, 2005) and the strain of mice (Belknap et al, 1998; Patti et al, 2005).

The result of our brain immunohistochemical study of $\mathrm{PKCI} / \mathrm{HINT} 1$ revealed that the protein is found in various regions including mesocorticolimbic and mesostriatal terminal regions; and is primarily localized to neurons, which is consistent with the result of in situ hybridization analysis of HINT1 mRNA (Vawter et al, 2004). A previous study on the intracellular localization of PKCI/HINT1 protein revealed that $\mathrm{PKCI} / \mathrm{HINT} 1$ was present mainly in the nucleus with lesser amount in the cytoplasm. However, that intracellular localization study (Klein et al, 1998) was carried out on non-neuronal cells. Our cellular distribution studies in neuronal cells have shown that mPKCI/HINT1 is primarily localized in the cytoplasm and neural processes. The possibility exists that the differential subcellular distribution in neuronal $v s$ non-neuronal cells reflects differential functions for this protein in nervous $v s$ peripheral tissues.

Finding the potential functional phenotype of a PKCI/ HINT1 deficiency is also of significant clinical relevance, as this protein has been one of the candidate protein molecules whose expression is altered in schizophrenic patients (Vawter et al, 2001). PKCI/HINT1 was identified as one of the downregulated genes in brain samples of schizophrenic patients (Vawter et al, 2004). The observation that PKCI/ HINT1 deficiency results in increased sensitivity to apomorphine and AMPH psychostimulant effects is consistent with an involvement of this protein in at least some of the symptoms of schizophrenia. Thus, the present results suggest that further research in the role of this protein on the pathophysiology of schizophrenia is clearly warranted. Several interesting questions arise for future research. An important issue is how the dopaminergic function is affected by PKCI/HINT1 and if the dopaminergic system is the only one to be involved. In addition it would be of interest to determine whether the PKCI/HINT1 KO mice could constitute an appropriate genetic model for schizophrenic research. It is recognized that there are some limitations in using conventional $\mathrm{KO}$ mice in studying the specific functions of a gene. Thus, the presence of a gene alteration in all the tissues naturally expressing the gene raises problems when attempting to assign a behavioral alteration to a specific brain structure or a pathway. Moreover, compensatory changes in other genes could occur in animals after genetic alteration. Therefore, using additional and complementary approaches to verify and further examine the function of PKCI/HINT1 in CNS would be required in order to gain a better understanding of PKCI/ HINT1 protein and its role in normal brain function and schizophrenia.

In conclusion, this study has revealed an interesting neurological phenotype, the supersensitivity to AMPH, in PKCI/HINT1 KO mice. It has also provided evidence to suggest that DA postsynaptic transmission is the primary site affected by the lack of the PKCI/HINT1 gene in mice. These results suggest an important role of PKCI/HINT1 protein in CNS function, especially for DA neurotransmission, modulating basal locomotor activity and the behavioral response to AMPH.

\section{ACKNOWLEDGEMENTS}

Support for this work was provided in part by grants from NIDA/NIH to JB Wang (DA11925, DA018722) and AS Undie (DA17614). We thank Dr IB Weinstein from Columbia University for providing breeding pair of $\mathrm{KO}$ mice and $\mathrm{Ms}$ $\mathrm{N} \mathrm{Wu}$ for the excellent technical support in genotyping.

\section{REFERENCES}

Abi-Dargham A, Rodenhiser J, Printz D, Zea-Ponce Y, Gil R, Kegeles LS et al (2000). Increased baseline occupancy of D2 
receptors by dopamine in schizophrenia. Proc Natl Acad Sci USA 97: 8104-8109.

Baldessarini RJ, Tarazi FI (2006). Pharmacotherapy of psychosis and mania. In: Brunton LL, Lazo JS, Parker KL (eds). Goodman and Gilman's the Pharmacological Basis of Therapeutics. McGraw-Hill: New York, Chicago. pp 461-500.

Belknap JK, Riggan J, Cross S, Young ER, Gallaher EJ, Crabbe JC (1998). Genetic determinants of morphine activity and thermal responses in 15 inbred mouse strains. Pharmacol Biochem Behav 59: 353-360.

Brzoska PM, Chen H, Zhu Y, Levin NA, Disatnik MH, MochlyRosen D et al (1995). The product of the ataxia-telangiectasia group D complementing gene, ATDC, interacts with a protein kinase C substrate and inhibitor. Proc Natl Acad Sci USA 92: 7824-7828.

Chefer VI, Shippenberg TS (2006). Paradoxical effects of prodynorphin gene deletion on basal and cocaine-evoked dopaminergic neurotransmission in the nucleus accumbens. Eur J Neurosci 23: $229-238$.

Giros B, Jaber M, Jones SR, Wightman RM, Caron MG (1996). Hyperlocomotion and indifference to cocaine and amphetamine in mice lacking the dopamine transporter. Nature 379: 606-612.

Guang W, Wang H, Su T, Weinstein IB, Wang JB (2004). Role of mPKCI, a novel mu-opioid receptor interactive protein, in receptor desensitization, phosphorylation, and morphine-induced analgesia. Mol Pharmacol 66: 1285-1292.

Halberda JP, Middaugh LD, Gard BE, Jackson BP (1997). DAD1and DAD2-like agonist effects on motor activity of C57 mice: differences compared to rats. Synapse 26: 81-92.

Herz A (1998). Opioid reward mechanisms: a key role in drug abuse? Can J Physiol Pharmacol 76: 252-258.

Klein MG, Yao Y, Slosberg ED, Lima CD, Doki Y, Weinstein IB (1998). Characterization of PKCI and comparative studies with FHIT, related members of the HIT protein family. Exp Cell Res 244: 26-32.

Lewis DA, Lieberman JA (2000). Catching up on schizophrenia: natural history and neurobiology. Neuron 28: 325-334.

Lima CD, Klein MG, Hendrickson WA (1997). Structure-based analysis of catalysis and substrate definition in the HIT protein family. Science 278: 286-290.

Lima CD, Klein MG, Weinstein IB, Hendrickson WA (1996). Three-dimensional structure of human protein kinase $\mathrm{C}$ interacting protein 1, a member of the HIT family of proteins. Proc Natl Acad Sci USA 93: 5357-5362.

McDonald JR, Walsh MP (1985). Inhibition of the $\mathrm{Ca}^{2+}$ - and phospholipid-dependent protein kinase by a novel $\mathrm{Mr} 17000$ $\mathrm{Ca}^{2+}$-binding protein. Biochem Biophys Res Commun 129: 603-610.
Namkung Y, Sibley DR (2004). Protein kinase C mediates phosphorylation, desensitization, and trafficking of the D2 dopamine receptor. J Biol Chem 279: 49533-49541.

Patti CL, Frussa-Filho R, Silva RH, Carvalho RC, Kameda SR, Takatsu-Coleman AL et al (2005). Behavioral characterization of morphine effects on motor activity in mice. Pharmacol Biochem Behav 81: 923-927.

Sanberg PR, Zoloty SA, Willis R, Ticarich CD, Rhoads K, Nagy RP et al (1987). Digiscan activity: automated measurement of thigmotactic and stereotypic behavior in rats. Pharmacol Biochem Behav 27: 569-572.

Schenk JO (2002). The functioning neuronal transporter for dopamine: kinetic mechanisms and effects of amphetamines, cocaine and methylphenidate. Prog Drug Res 59: 111-131.

Seeman P (1987). Dopamine receptors and the dopamine hypothesis of schizophrenia. Synapse 1: 133-152.

Segal DS, Geyer MA, Schuckit MA (1981). Stimulant-induced psychosis: an evaluation of animal methods. Essays Neurochem Neuropharmacol 5: 95-129.

Smith AD, Justice JB (1994). The effect of inhibition of synthesis, release, metabolism and uptake on the microdialysis extraction fraction of dopamine. J Neurosci Methods 54: 75-82.

Spanagel R, Herz A, Shippenberg TS (1992). Opposing tonically active endogenous opioid systems modulate the mesolimbic dopaminergic pathway. Proc Natl Acad Sci USA 89: 2046-2050.

Su T, Suzui M, Wang L, Lin CS, Xing WQ, Weinstein IB (2003). Deletion of histidine triad nucleotide-binding protein 1/PKCinteracting protein in mice enhances cell growth and carcinogenesis. Proc Natl Acad Sci USA 100: 7824-7829.

Sulzer D, Maidment NT, Rayport S (1993). Amphetamine and other weak bases act to promote reverse transport of dopamine in ventral midbrain neurons. J Neurochem 60: 527-535.

Vawter MP, Barrett T, Cheadle C, Sokolov BP, Wood III WH, Donovan DM et al (2001). Application of cDNA microarrays to examine gene expression differences in schizophrenia. Brain Res Bull 55: 641-650.

Vawter MP, Crook JM, Hyde TM, Kleinman JE, Weinberger DR, Becker KG et al (2002). Microarray analysis of gene expression in the prefrontal cortex in schizophrenia: a preliminary study. Schizophr Res 58: 11-20.

Vawter MP, Shannon WC, Ferran E, Matsumoto M, Overman K, Hyde TM et al (2004). Gene expression of metabolic enzymes and a protease inhibitor in the prefrontal cortex are decreased in schizophrenia. Neurochem Res 29: 1245-1255.

Weiske J, Huber O (2005). The histidine triad protein Hint1 interacts with Pontin and Reptin and inhibits TCF-beta-cateninmediated transcription. J Cell Sci 118: 3117-3129. 